\title{
Efficient and sustainable energy lighting solutions
}

\section{Angel García-Botella, Daniel Vázquez-Moliní, Berta Garcia-Fernandez, Antonio-Álvarez Fernandez-Balbuena}

Angel García-Botella, Daniel Vázquez-Moliní, Berta Garcia-Fernandez, Antonio-Álvarez Fernandez-Balbuena, "Efficient and sustainable energy lighting solutions," Proc. SPIE 11120, Nonimaging Optics: Efficient Design for Illumination and Solar Concentration XVI, 111200Q (9 September 2019); doi: $10.1117 / 12.2529187$

SPIE Event: SPIE Optical Engineering + Applications, 2019, San Diego, California, United States 


\title{
Efficient and sustainable energy lighting solutions
}

\author{
Angel García-Botella ${ }^{1}$, Daniel Vázquez-Moliní ${ }^{2}$, Berta Garcia-Fernandez ${ }^{12}$, Antonio Álvarez \\ Fernandez-Balbuena ${ }^{1}$, \\ ${ }^{1}$ Departamento de Ingenieria y Gestion Forestal y Ambiental, Universidad Politécnica de Madrid, ETSI de \\ Montes. Ciudad Universitaria s/n 28040 Madrid (SPAIN). e-mail: angel.garciab@upm.es \\ ${ }^{2}$ Departamento de Óptica, Universidad Complutense de Madrid, Fac de Óptica y Optometría. Arcos de Jalón, \\ 118, 28037 Madrid (SPAIN).
}

\begin{abstract}
The electric lighting is responsible for $15 \%$ of the electricity consumption, the efficiency in this sector has a good progress in recent years, it can still improve more. Conventional energy sources are polluting and limited, so it is essential to use less aggressive energies with the environment. In lighting, this premise is being implemented in a cross-cutting manner, but in response to varied and sometimes contradictory interests. The development of lighting devices based on solar radiation is an inalienable objective, as is the use of artificial lighting sources that are clean with the environment. To achieve these two objectives, it is essential to know and study the design techniques of non-image optics. In the present work we present new systems design techniques and lighting elements: ray tracing optimization technique, light vector field technique. As well as its implementation and application in various areas of lighting: lighting in office buildings, libraries museums. We show that renewable energy strategy in the field of lighting not only produce energy save, also produce good effects in other aspects of human life like well-being, productivity and heritage conservation.
\end{abstract}

\section{INTRODUCTION}

Communities across the world are living a transition to 100\% Renewable Energy (RE) in energy supply conditioned by the importance of caring for the planet. Solar energy is the most important RE source and represents an optimal alternative to produce electricity and illuminate buildings. Almost 15\% of produced electricity is being consumed for lighting in all over the world [1]

Demand for lighting is continued increasing, driven by strong growth in many fields like building floor area and back lighting for displays. Within this context, improving energy saving is essential by means of efficient lighting systems, such as natural illumination and artificial lighting based on LED (Light Emitting Diode) [2]. Natural lighting is conditioned by geographic location and climatology so that it needs specific control strategies of design and exhaustive studies of each particular situation. In this sense, advantages of nonimaging optics including light guides, nonimaging reflectors, lenses or a combination of both systems offers energy saving opportunities over existing artificial lighting. LED market has experienced terrific growth recently and this remarkable trend continue because is one of today's most energy-efficient lighting system. In this work we present a review about daylighting and artificial lighting applications and actuations developed with the objective of renewable energy implementation in the field of lighting, conclusions about more than twenty years expertise on the developments of energy efficient lighting systems will be given.

\section{ADVANCED TOOLS FOR ILLUMINATION SYSTEMS DESIGN}

Illumination systems design is usually included in what have been called "nonimaging optics", at the same time it is in the area of photometry and governed by the laws of geometrical optics. Several techniques have been developed during the recent years (SMS method, differential 
equations methods) [3] all of them focused in the ray propagation analysis. In this introduction we focus our attention in two powerfull techniques Raytrace Optimization techniques and Flow line vector, which has showed great potential to overcome classical results in the field of lighting design. By one side the incorporation of genetic and evolutionary algorithms in optimization procedures has provide a new view in the global optimization problems and on the other hand the use of vector field theory in the geometrical optics laws also provides new perspective to the nonimaging optical systems design.

\subsection{AUTOMATIC RAYTRACE OPTIMIZATION}

Automatic optimization techniques have been introduced in the design of nonimaging systems recently [4]. Nonsequential raytrace and complex non centred optical systems with, large ray number, are the main impediment to extensive application of automatic optimization techniques to nonimaging optics problems. Nowadays, improvements in hardware and software capabilities allows the implementation of optimization utilities in software packages, becoming a powerful tool in the nonimaging design problem. The main steps in the nonimaging optimization procedure are: first the parameterization of optical system, including the definition of the constraints in the parameters, second the definition of the merit function to be minimized or maximized and third the selection of the optimization algorithm. There are numerous optimization algorithms that can be used in nonimaging optics optimization, including simplex method, polynomial fitting, global synthesis method and evolutionary algorithm [5] in particular simplex algorithm produces robust, lower consumption and convergent method in nonimaging optimization problem [6].

Commercial raytrace software package include optimization tools, but those tools nowadays are under development, and not cover completely the needs for global optimization of illumination systems. We propose a fundamental methodology, using basic CAD software (Catia, Solidworks,...) also a raytracer (TracePro, Lightools...) and control and analysis of the procedure and the results by Matlab software. This work scheme improves the potentiality and capability of the optimization technique. Allow us to control all the geometrical parameters, also materials properties, we can analyse all photometrical parameters (efficiency, uniformity, light intensity...) we can modify and study the right Merit Function and finally we can choose the optimization algorithm supported by Matlab.

We focus our attention in the merit function, as it has the role to drive the optimization procedure. Then, improvements in the capabilities of merit function will improve the results of the optimization procedure. The most common way to build merit functions involves the weighted sum of squares of the differences between a set of objectives and their associated target values:

$$
M F=\sum_{i} w_{i}\left(V_{i}-T_{i}\right)^{2},
$$

\section{(1)}

where $w_{i}$ is the weight factor for the $i^{\text {th }}$ objective, $V_{i}$ is the value of the $i^{\text {th }}$ objective and $T_{i}$ is the target value of the $i^{\text {th }}$ objective. Equation (1) shows direct influence of the weight factors in the $M F$ and therefore in the optimization procedure. Commonly the weights factors $\left\{w_{i}\right\}$ are manually adjusted by trial and error procedure [9], this non-optimal situation suggest the need to study methods for automatic adjustments of the weight factors $\left\{w_{i}\right\}$. In this paper we propose a new type of merit functions, Dynamic Merit Functions (DMF), which automatically adjust the weight factors $\left\{w_{i}\right\}$ during the progress of optimization procedure. The variation of weight factors modifies the optimization problem, and $D M F$ becomes a global optimization method. 
We have checked $D M F$ algorithm for several standard nonimaging systems [7]. Figure 1 shows the results for uniformizing lens and flat LED luminaire improving about $25 \%$ the efficiency $(\Delta \eta)$ and $10 \%$ of mean uniformity improvement $(\Delta \mathrm{U})$, in comparison with $C M F$ (Conventional Merit Function). We have also analyzed the dependence of the DMF optimization method with number of variable parameters in the nonimaging optical system. We can conclude that $D M F$ provides better results for systems with greater number of parameters, which means that $D M F$ is a right method to design complex optical systems.
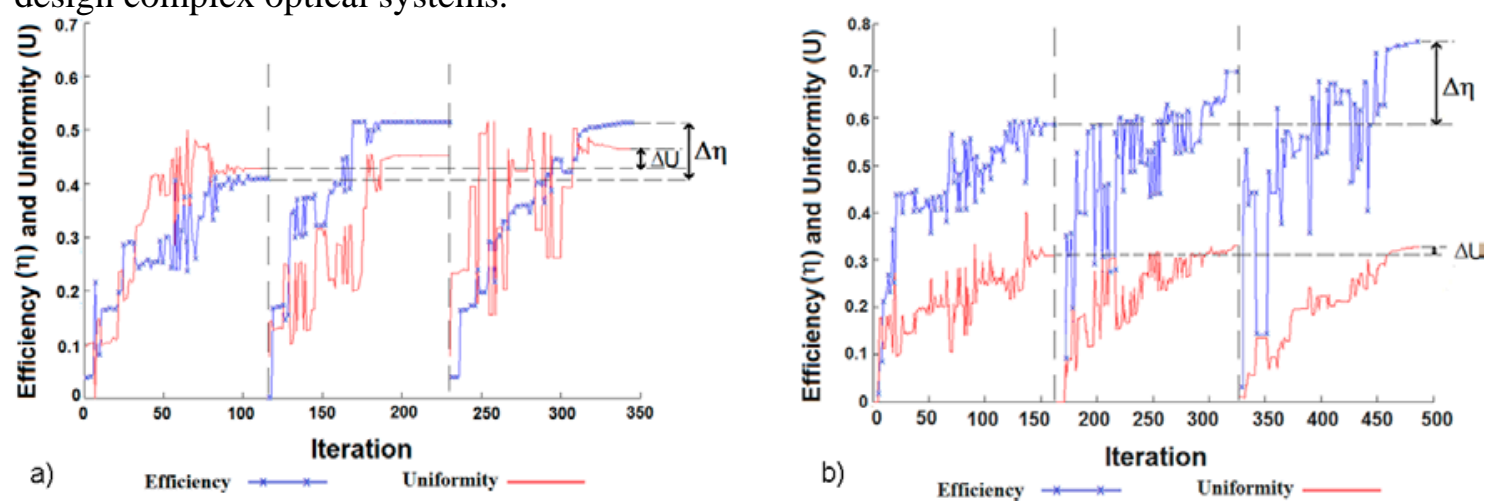

Fig.1 a) DMF applied to uniformizing lens, vertical dashed lines indicate the adjust in the weight factors $\left(w_{i}\right)$ b) DMF applied to flat LED luminare, also vertical dashed lines indicate the adjust in the weight factors

\subsection{FLOW LINE VECTOR DESIGN METHOD}

The Flow line method, which starts with the definition of the irradiance vector $\mathbf{J}$, is one of the techniques used in nonimaging optics. Called "Geometrical flux vector" it has provided ideal designs. The main property of this model is its ability to estimate how radiant energy is transferred by the optical system, from the concepts of field line, flux tube and vector potential, overcoming traditional raytrace methods. Nevertheless this model has been developed only at an academic level, where characteristic optical parameters are ideal not real and the studied geometries are simple. The main objective of the present paper is the application of the vector field method to the analysis and design of real concentration and illumination systems. We propose the development of a calculation tool for optical simulations by vector field, using algorithms based on Fermat`s principle, as an alternative to traditional tools for optical simulations by raytrace, based on reflection and refraction law. This new tool provides, first, traditional simulations results: efficiency, illuminance/irradiance calculations, angular distribution of light- with lower computation time, photometrical information needs about a few tens of field lines, in comparison with million rays needed nowadays. On the other hand the tool will provides new information as vector field maps produced by the system, composed by field lines and quasipotential surfaces.

The flowline method was introduced by Winston and Welford [8], based on the concept of geometrical flux vector $\mathbf{J}$, from the application of the etendue conservation law for a loss-free optical system. The components of the geometrical flux vector are

$$
J_{x}=\int d p_{y} d p_{z}, \quad J_{y}=\int d p_{x} d p_{z}, \quad J_{z}=\int d p_{x} d p_{y}
$$

where $d p_{x}, d p_{y}$ and $d p_{z}$ are the direction cosines or, for more general considerations, the generalized momenta in phase space. The physical meaning of geometrical flux vector is that, the $J_{z}$ component, for instance, is proportional to the total flux per unit area entering the $x, y$ plane. The most important property of flowline method is that a way to construct concentrators with maximum concentration ratio is to place mirrors with the geometry of flowlines. In an analogous way Gershun [9], introduced the concept of light vector, the magnitude of light vector is equal to the net radiant power per unit area at point $P$, and the direction of the vector is the direction of the flow of the radiant energy at that point. To analyze 3D optical systems we will use the concept of vector 
potential of this light vector, in way that geometrical flux vector can be computed from vector potential $\mathbf{A}$, as

$$
\mathbf{J}=\operatorname{curl} \mathbf{A}
$$

where

$$
\boldsymbol{A}=\frac{H}{2} \oint \ln r \boldsymbol{d} \boldsymbol{l}
$$

where $r$ is the distance between the contour of the source and any point $P$ in the space. The integral is calculated for the closed contour of light source, $\boldsymbol{d} \boldsymbol{l}$ is an infinitesimal vector through the direction of the contour of the light source and $H$ is the luminance/radiance of the source, constant for Lambertian radiators and we can get it out of the integral. For refractive and reflective systems we will replace the distance by optical path length [10].

As main objective it is possible to identify the need to use global algorithms to evaluate the geometric flux vector from vector potential computations (eq.4) at each point in the space. It is necessary an algorithm able to be used in 2D and 3D calculations, able to study reflexive, refractive and diffuse materials, able to study systems with $\mathrm{N}$ optical surfaces. In fact it must be a fundamental algorithm to cover all possible configurations.

We start the task to build simulation software, to analyze the optical behavior of systems based on the calculus of irradiance vector field. We start that task using MATLAB software and our objective is to use GUI (Graphical User Interface) toolbox to provide an easy to use software interface. Of course this first version of the software includes big constraints, which will be removed step by step. First one is that simulation software only analyzes 2D systems with a source, a lens/mirror and a detector. The dimensions of the source lens and detector are constrained too, in order to ensure the reliability of the software. With these constrains we have built an interface which includes fields to design the systems and three main capabilities, first one draw the system, second ones simulates the irradiance map at detector third simulates field lines in the space. Figure 2 shows the interface of the software, we have uploaded a free version of the VF_simulator in our webpage http://www.ucm.es/lightingandcolor.

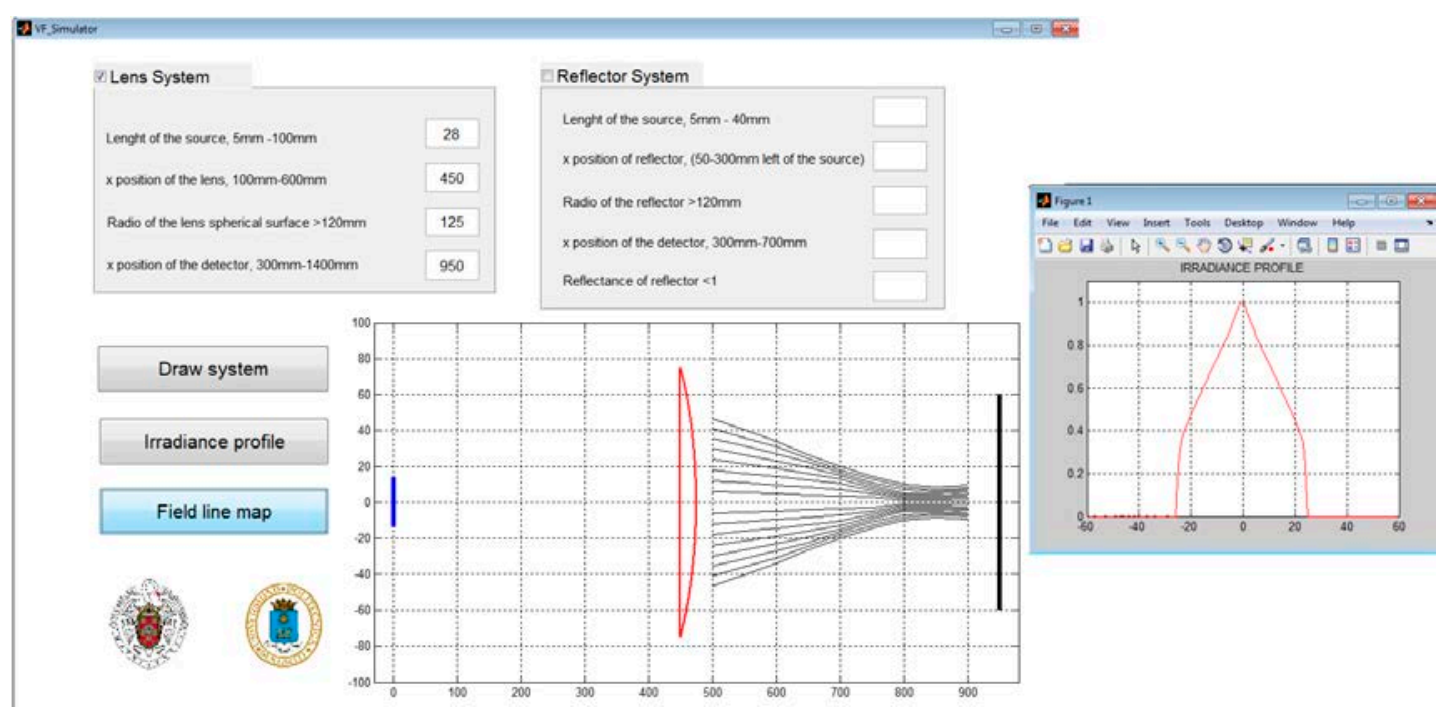

Fig.2 Flowline based optical simulation software interface, including calculated field map and irradiance profile. Free version of this software is available at http://www.ucm.es/lightingandcolor 


\section{ENERGY SAVING LIGHTING SYSTEMS}

Buildings are responsible for nearly $40 \%$ of primary energy consumption, half of electricity consumption, and about a third of greenhouse gas emissions. In this situation, appropriate daylighting design is required, to ensure that the overall use of energy is minimized [11].

The widespread use of daylight lighting coupled with the use of the most energy efficient lighting technologies, such as LEDs or lighting controls reduces the energy consumption of electricity. The use of daylighting has many advantages such as: energy savings reducing need for artificial light, occupant well-being improving performance of workers and therefore producing an increase of productivity and comfort [12].

The economic costs and benefits of using natural lighting vary greatly depending on facts like the geographical location and the configuration of the optical system used [13]. Their calculation should include parameters that can be easily quantified as the cost of materials employed but others as the climatology or those related to the comfort and well-being of the occupants are difficult to estimate. The doubtless fact is that taking advantage of natural lighting is a guarantee to improve the well-being of occupants as well as saving energy.

\subsection{DAYLIGHT UTILIZATION FOR BUILDINGS}

The overall objective of daylighting is to minimize the amount of artificial light and reduce electricity costs, Electrical lighting produces a lot of heat and studies suggest that daylighting has a direct impact on well-being, productivity and overall sense of satisfaction.Within natural lighting systems, tubular daylighting systems called light pipes or light tubes are developments to increase daylighting in interior spaces of buildings where contribution of natural light is minimal or absent. Light guides are considered one of the key renewable energy sources used in daylight transporting light long distances by reflections on their surfaces [14].-The guide of light is designed according to many parameters like efficiency and health ambient but the use a light guide is not usually supported. One drawback is that often it is necessary long distance to reach and some characteristics of the materials used, such as absorption of the material limit the guided. Another drawback is due to the space limitations and position of the objects to be illuminated. In some cases is necessary to bend the light guide resulting in a decrease in efficiency and uniformity. This section presents several developments adapted to buildings to solve those inconveniences.

\subsubsection{DAYLIGHT ENHANCEMENT SYSTEM}

In this section we presents an optical design that takes advantage of solar energy reaching the facade of the building for lighting purposes reducing building energy consumption called ADASY. This daylighting system's structure comprises a light collector located outside the vertical facade of the building, a modular light guide that runs along the horizontal false ceiling and light extractor luminaires strategically positioned along the guide (Fig. 3). The system is configured specifically to the particular characteristics of each building (facade orientation, latitude and longitude) to optimize the global performance by the anidolic collecting system. The daylighting system has been completely developed and experimentally tested, and its prototype is currently integrated into Lledó S.A company's facilities [15]. 


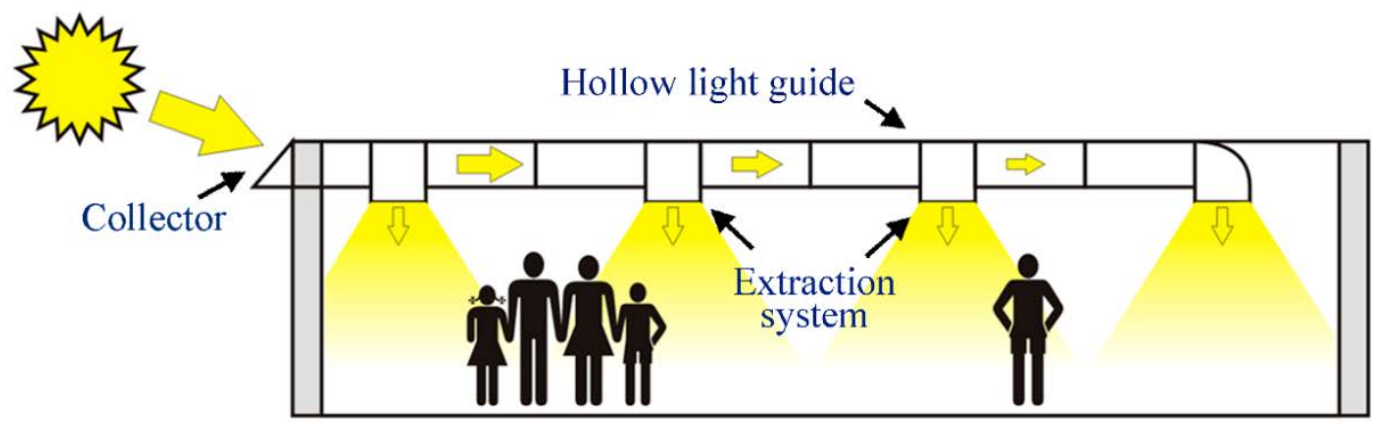

Fig.3 Daylighting system's structure: light collector located outside the vertical facade of the building, a modular hollow light guide and light extractor luminaires.
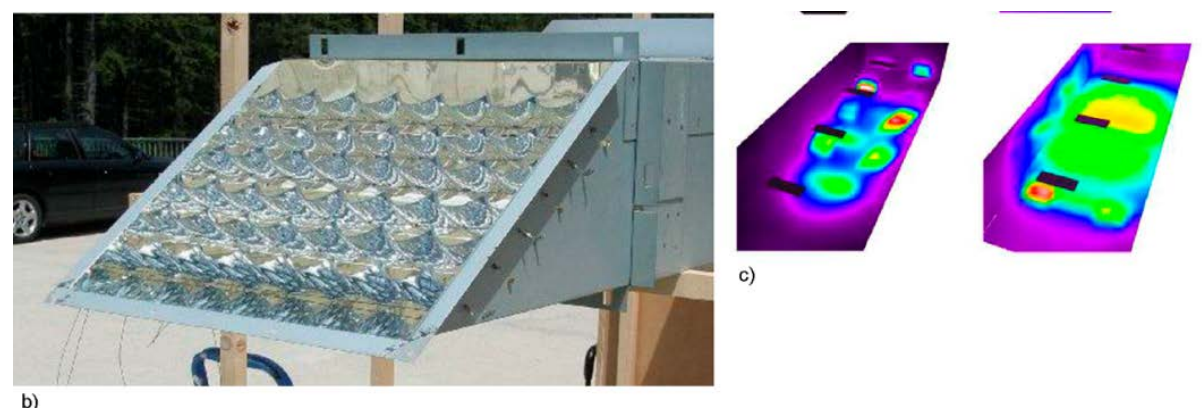

Fig 4 Parabolic component called T-CPC (Compound Parabolic Concentrator Truncated) (a) that make up the collector structure (b) the flux is redirected to room (c).

The collecting system, based on anidolic optical components, is responsible to collect and redirect sunlight minimizing solar incidence angle dependence (Fig.4 (a)). The collector structure is based on the parabolic component called T-CPC (Compound Parabolic Concentrator-Truncated) (Fig.4 ( b)) which reflect all the incident radiation within wide limits, increasing the exploitable incident radiation. Once the incident sunlight is captured, the flux is redirected to room zones distant from the window where the sunlight can't reach directly. This is accomplished by a horizontal light guide inside building's false ceiling. The light is channeled inside these high reflectivity mirrored devices through reflection on their inner faces. Finally a system for light extraction/distribution had been devised to spread the sunlight efficiently and balanced into the room as shown figure 3 (c). The cost-effective components of the system and its adaptable features make ADASY a practical, lowmaintenance daylight solution that can easily be integrated into nonresidential buildings. This system can help architects to reduce electrical illumination a sunlight collection device for solar lighting systems aimed at non-residential buildings.The whole system provides a lighting level of 300-400 lx during the mid-time work hours in an area of $4 \mathrm{~m} \times 10 \mathrm{~m}$, i.e. $40 \mathrm{~m}^{2}$. The sun-luminaires manage to reduce glare (interference with vision or eyestrain) while maintaining an optimal distribution, providing interesting solutions for office buildings.

\subsubsection{DAYLIGHTING DESIGN FOR LIBRARY INSTALLATIONS}

Another interesting application of Daylight was developed for libraries building. Considering that reading is the main function of use in libraries, proper lighting becomes a crucial factor in the overall success of a library design. With this proposal we offers new insights into a case study providing an efficient lighting design for library installations. After examine the conditions of indoor daylight and the library's energy performance in the faculty of Architecture, Design and 
Built Environment, Beirut Arab University with architectural elements as space depth, window size, external obstruction angle, and glazing visible transmittance [16]. In this case, the insufficient amount of natural daylight within the space itself, produces a high consumption of artificial light during the day, hence consuming higher amounts of energy. The solution to-this problem, due to the geographical location of the building and the proximity of the interior space to the roof of the building, is the incorporation of light guides, in particular, by vertical hollow prismatic light guides. Tubular guides can transfer natural light from a building's roof into the depths of the building. Hollow Prismatic Light Guides (HPLGs) offer a particular technical alternative within tubular guides, they are internally covered with a thin and transparent prismatic sheet. The HPLGs leads light beams by Total Internal Reflection (TIR) and provides light transmission with high efficiency, minimal colour shifts and homogeneous light distribution [17]. Two specifics designs of HPLGs developed according to the dimensions of the spaces act as luminaires to increase efficiency and uniformity distribution of natural light into the library spaces (Fig. 5). Tridimensional realistic simulations had been carried out by prismatic film parameters according with the commercially film OLF [18] with a non-sequential optical ray tracing software called TracePro 7.4 [19] additionally defects in prismatic corners has been set to be a corner radii of $3 \mu \mathrm{m}$ [17]. Two designs are configured allowing achieve high illumination levels in working plane by an optimization procedure. For this application, three holographic diffusers of plastic with Gaussian diffusion functions from Luminit Optics [20] were chosen according to the scene and optical requirements of guiding. The transparent diffuser sheet called Luminit 30x5 degree LSD (called D1) is located at the input of each guide and the dimensions agree with the diameter of the guide. The input diffuser D1 emits in a semi-angle of $30^{\circ}$ which approximately maintains the maximum acceptance angle determined by the refractive index of the prismatic film. This sheet disperses the light and provides a luminous distribution curve determined by a Bidirectional Transmittance Distribution Function (BTDF) allowing to obtain an optimal angular distribution to internally reflect the light beam optimizing to the maximum light transmittance in the system. Several diffusers are placed at the end of the models, providing an improvement in light distribution improving uniformity in the work plane of the library's tables. Model 1 is composed of Luminit 60 degree LSD (called D2) plus Luminit 40 degree LSD (called D3). D2 is a centered octagon with a side length of $0.099 \mathrm{~m}$ and D3 is a side square of $0.44 \mathrm{~m}$. Model 2 is composed of Luminit 60 degree LSD (D2). Figure 1 shows a detail of dimensions, in model $1 \mathrm{a}=0.5 \mathrm{~m}, \mathrm{~b}=0.01 \mathrm{~m}$ and $\mathrm{c}=0.07 \mathrm{~m}$, in Model $2 \mathrm{a}=0.55 \mathrm{~m}$ and $\mathrm{b}=0.175 \mathrm{~m}$.

The transmission efficiency $\left(\mathrm{T}_{\mathrm{g}}\right.$ ) used to characterize the flux of light transmitted through the guide, is defined as:

$$
\mathrm{T}_{\mathrm{g}}=\phi_{\mathrm{d}} / \phi_{\mathrm{I}}
$$

where $\phi_{\mathrm{d}}$ is the flux intercepted by the plane and $\phi_{\mathrm{i}}$ is the flux at the input of the guide. Transmission efficiency is evaluated at the end of the guides and in work plane. The dimensions used to characterize the guides are given as aspect ratio $(\rho)$ defined as length to diameter $(\rho=L / d)$.

The collimate emission pattern used to characterize the system simulate the sunlight distribution coming from a heliostat, which act as light collected system. Only one medium wavelength value is enough to set the light source due the characteristics of prismatic guide.

The luminous flux obtained in work plane of the library tables is shown in figures 8 and 9 . Figure 8 represents the flux ratio and irradiance map obtained in Model 1 and figure 9 represents the result obtained in Model 2. There is an increase of efficiency of Model 2 with regard to Model 1 mainly due to the smaller aspect ratio. In addition, Model 2 provides a more equitable distribution of light throughout the system. The illuminance map shows the flux received vs emitted (efficiency) and the profile plot in the horizontal and vertical axis. The measurements revealed a percentage of 
transmission efficiency of $64.7 \%$ in Model $1(\rho=15,60)$, and $78.82 \%(\rho=6,46)$ in Model 2 in work plane.

The illuminance uniformity in the work plane. U0, is calculated by:

$$
\mathrm{U}_{0}=\mathrm{E}_{\operatorname{med}} / \mathrm{E}_{\max }
$$

where $E_{\max }$ is the maximum value of the illuminance obtained and $E_{\text {med }}$ is the average value. The cropped area from $(-710,260)$ to $(710,-260)$ millimeters in figures 2 and 3 which corresponds to the work area has a high uniformity and has a $\mathrm{U}_{0}$ value of 0.49 in Model 1 and 0.54 in Model 2 . This system provides a high uniformity in working plane avoiding glare in users.
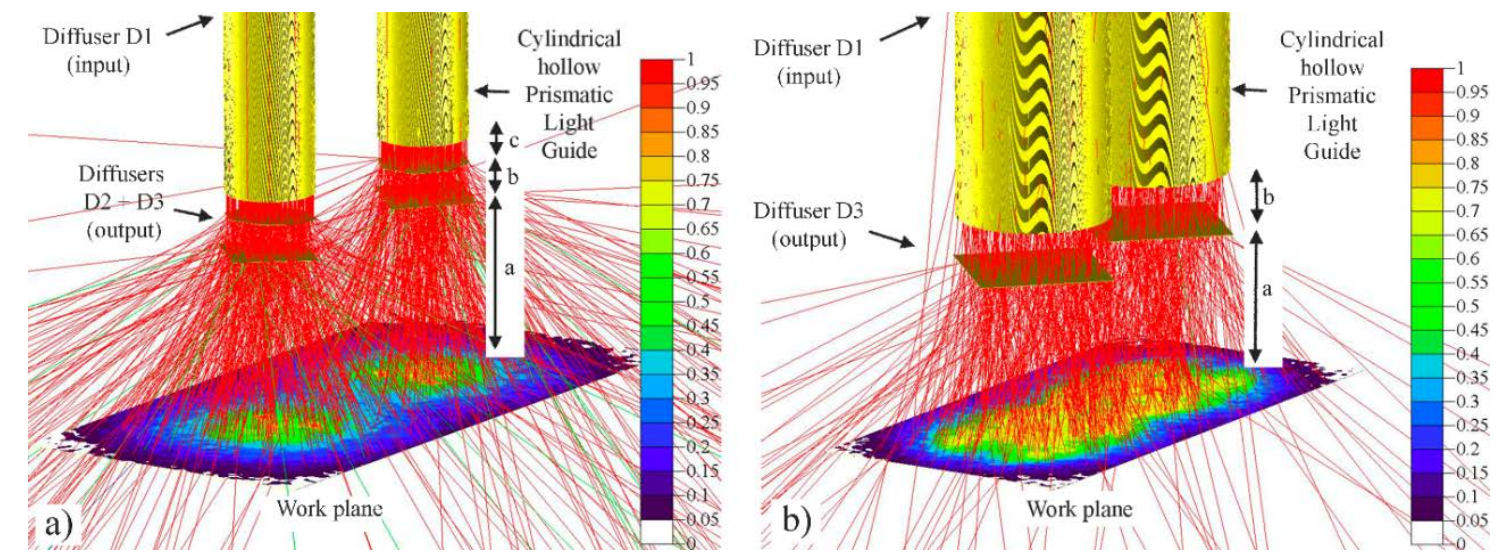

Fig 5. Tridimensional realistic raytrace simulations of HPLGs developed. Model 1 (a) and Model 2 (b).
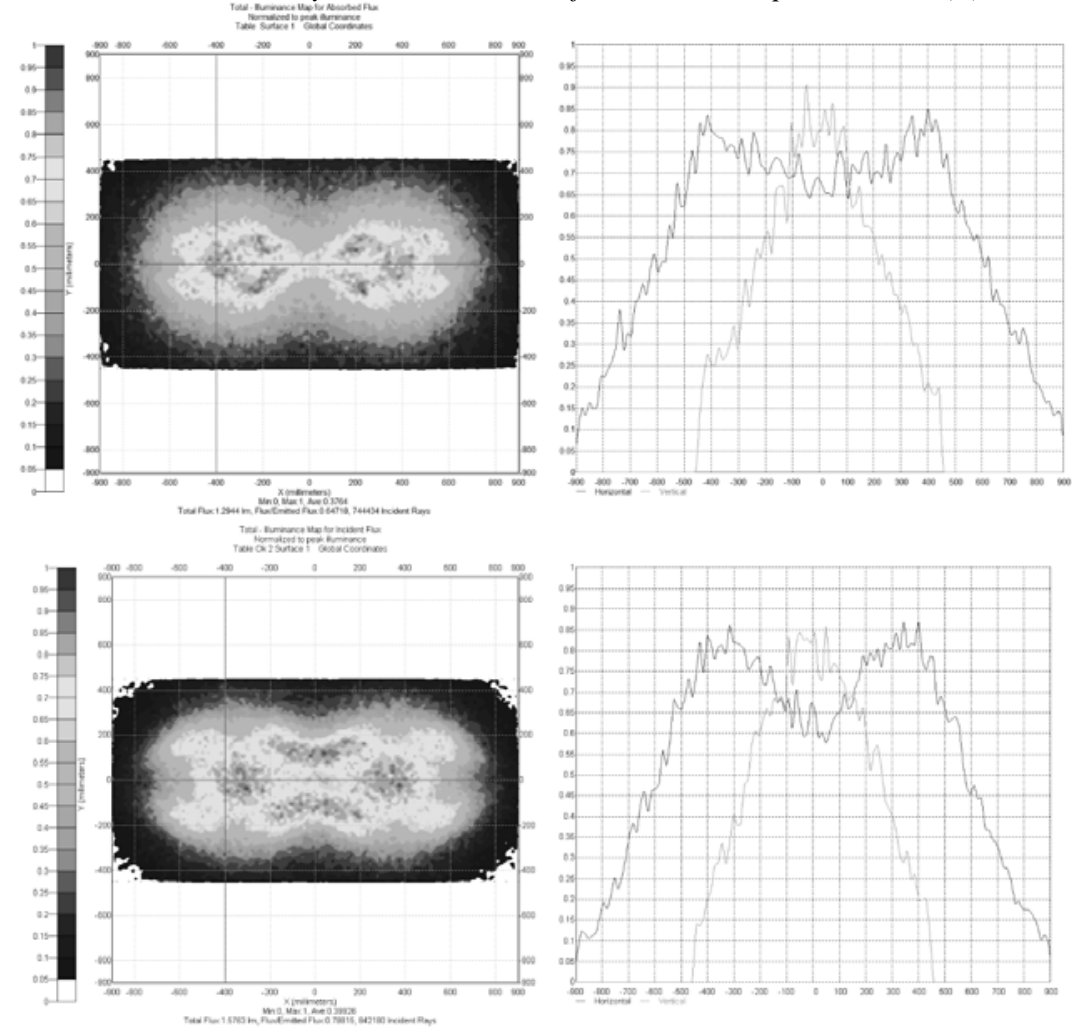

Fig 6. Illuminance map and horizontal and vertical profiles obtained in Model 1 with $\rho=15,6$ (upper) and Model 2 with $\rho=6,46$ (lower) on the working plane. 


\subsubsection{A DIRECTIONAL CUSTOMIZABLE BEND LIGHTING}

Light guide systems are fundamental elements in daylighting strategies. The standard design to guide the light commercially used in elbows is shaped by the rotation of the aluminum pieces with different angles. This geometrical configuration produces asymmetrical and inefficient spatial light distribution in the guidance system. To solve this problem, we design an elbow called Bend Lighting System (BLS) with two compound parabolic concentrators (CPCs) united by a standard elbow [20, 21], fig 7. A Theoretical analysis performed by realistic ray-tracing simulations is compared with an experimental development for a system by a $120^{\circ}$ BLS angular configuration. Results of light distribution by normalize illuminance measurement in theoretical (Fig. 8 (a)) and luminance in empirical system (Fig. 8 (b)) models are shows. This configuration allows illuminate spaces bending the light beams for a wide range of angular configurations with high efficiency. For a theoretical tridimensional model with a cylindrical guide diameter of $0.200 \mathrm{~m}$ and $3.5 \mathrm{~m}$ length results show a $T_{g}$ (eq. 3 ) of $82.3 \%$ at the exit of the HPLG $(\rho=17.35)$ and $70 \%$ at the exit of the BLS.
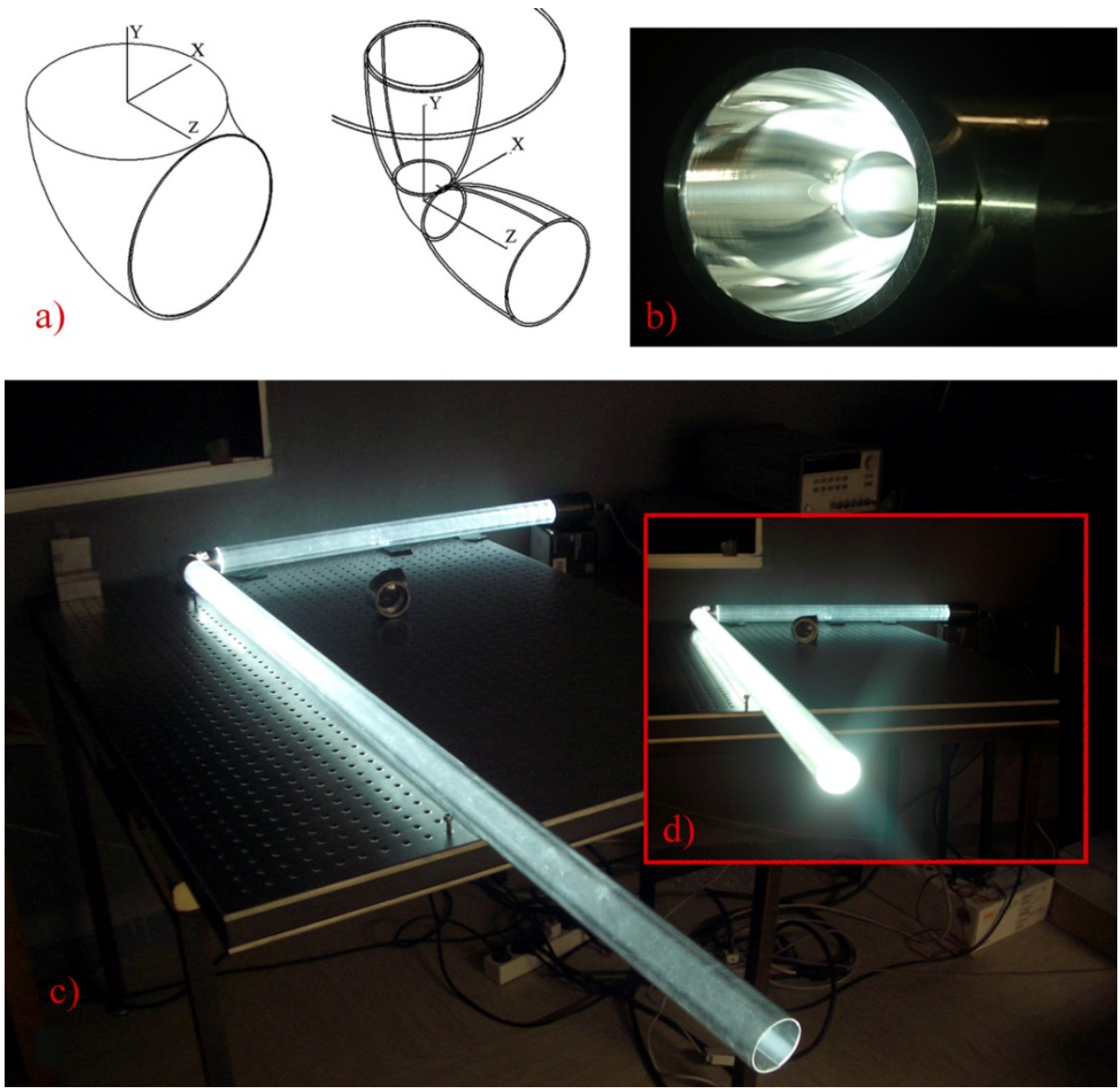

Fig.7Prototype of Bend Lighting System (BLS) with two compound parabolic concentrators 

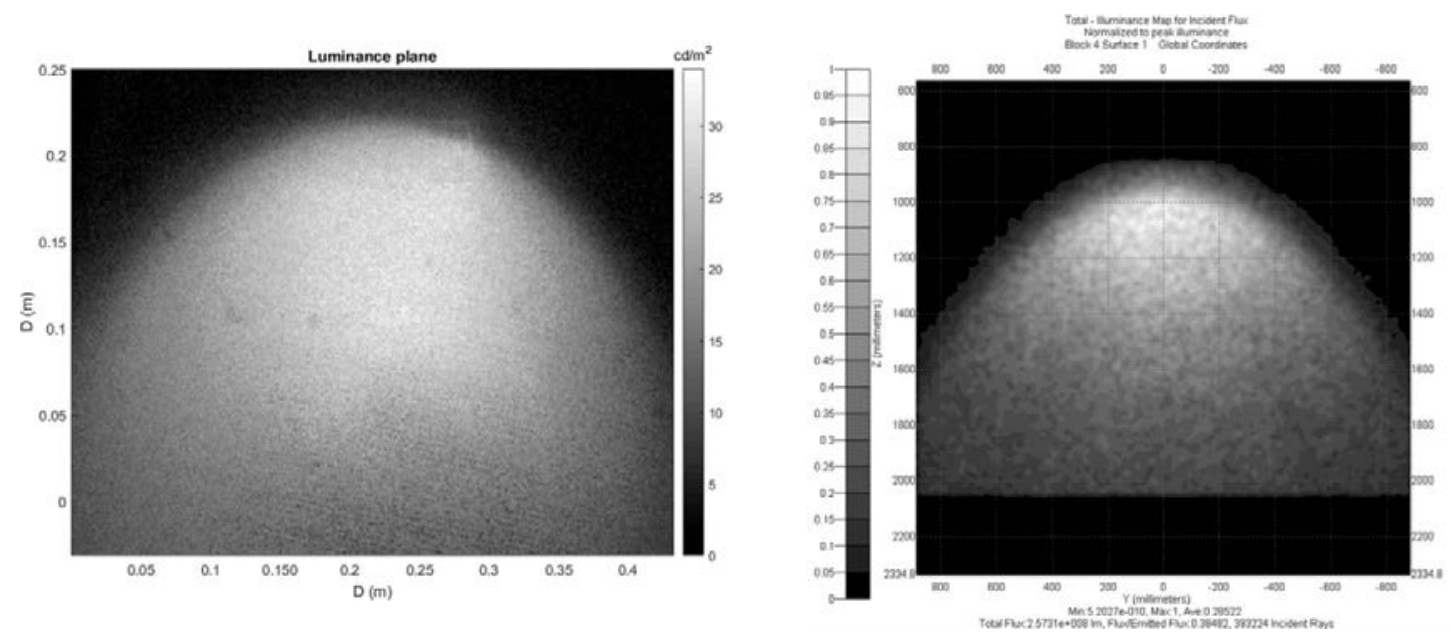

Fig.8 Illuminance map in output plane of theoretical measure, and luminance map in output plane of experimental measure.

\subsection{LED LIGHT PROJECTION FOR MUSEUM LIGHTING}

The use of new LED light sources allows new possibilities in the lighting design, as microelectronic components provides new control capabilities and at the same time accomplishing the objective of energy saving lighting systems. In this frame we have developed an active lighting system based on LED technology for museum lighting applications. The goal of this research is to develop light systems and sources with an optimized spectral distribution. The optimization implies to maximize the color fidelity reproduction and the same time to minimize the photochemical damage, those requirements of museum lighting are perfectly synchronized with our main objective of energy saving. In this way the perceived color under this sources will be similar (metameric) to technical requirements given by the restoration team uncharged of the conservation and exhibition of the goods of art. On the other hand, depending of the fragility of the exposed art objects (i.e. spectral responsivity of the material) the irradiance must be kept under a critical level. Therefore, it is necessary to develop a mathematical model that simulates with enough accuracy both the visual effect of the illumination and the photochemical impact of the radiation. Spectral reflectance of different reference painting ("The woman in blue" of Picasso and "Walk on the beach" of Sorolla) has been measured with a high resolution non.contact spectrophotometer [22], [23]. A light source has been modeled as four LEDs (red, green, blue and amber), those intensity will be varied from 0 to 100 . The mathematical model is based on a merit function that optimized the individual intensity of the LED-light sources taking into account the damage function of the material and color space coordinates. Moreover the algorithm used weights for damage and color fidelity in order to adapt the model to a specific museum application [22], [23].

The optimization algorithm must use two different information data: one is about colour reproduction and other is about damage. The system calculate, with required resolution, the colour and damage for all the points of the Paint. Depending on the type of light source used , its the spectral distribution can be modelled by parameters $\mathrm{k}$ : $\mathrm{k} 1, \mathrm{k} 2, \ldots, \mathrm{kn}$. Algorithm start with a specific spectral distribution which is defined by seed parameters. Then system calculates point by point, using the spectral reflectance the colour in CIELab coordinates, using the absorptance the damage associated with that illuminant. When all the points have been calculated then the algorithm uses weight parameters in order to evaluate how good is the calculated Merit Function. Figure 9 shows the application of this lighting system to the paintings. 


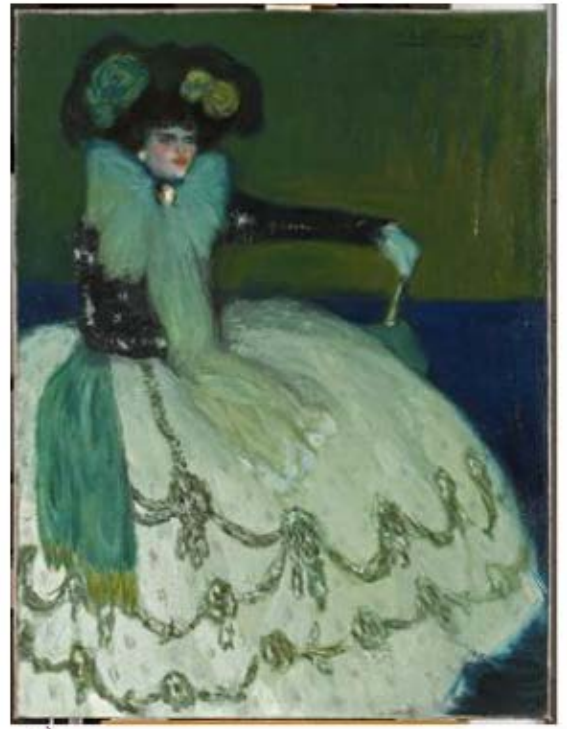

a)

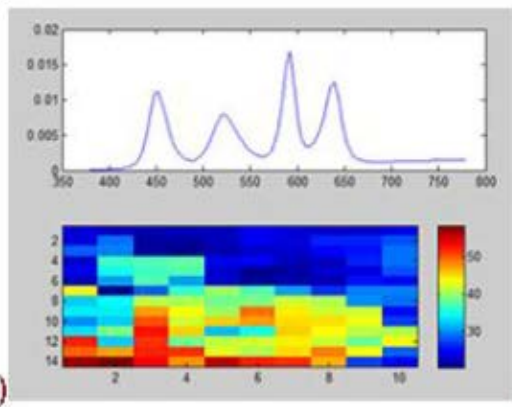

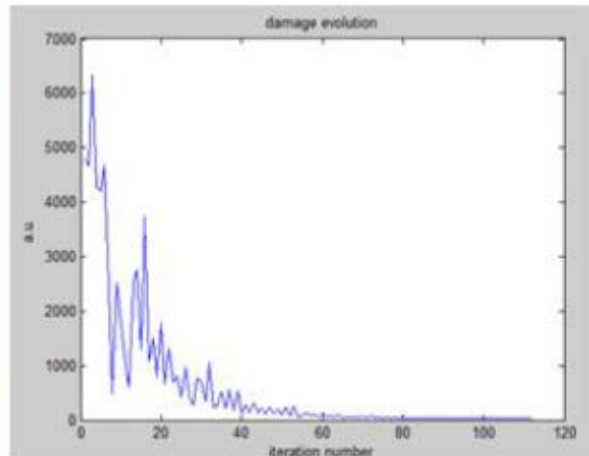

b)

d)

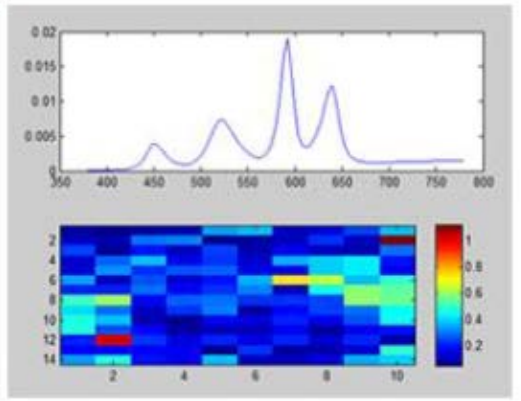

Fig.9 Application of point to point spectral selective lighting system to the "Woman in blue" of Pablo Picasso [22]

\section{CONCLUSIONS}

In this paper we have presented different actuations and applications developed during last years with the objective of renewable energy systems design in the field of lighting. We have developed new optical design techniques which can provides new efficient systems, Flowline method and optimization techniques are clear candidates to achieve that objective. In the objective of renewable energy implementation in the field of lighting it is essential the development of daylighting systems, even more taking into account the well-being and productivity properties of daylighting. We have presented several Daylighting application and implementations in cooperation with Spanish lighting companies and international institutions, achieving functional prototypes and actuations. The development of LED's sources provides new opportunities and it is also synchronized with the main objective of energy efficient implementation. In fact, as we have showed with the application of point to point spectral lighting LED projection for museum lighting, the renewable energy strategy is also synchronized with the objective of cultural heritage conservation by reducing the damage in cultural goods. All these expertise in applications and actuations provides a clear conclusion, it is essential to reinforce the renewable energy strategy, but not only to save energy also for other parameters related to human life like well-being, productivity and heritage conservation. In this sense, the research structures and the lighting industry in Spain are well prepared to advance in this strategy, what is needed is a clear commitment from the institutions. 


\section{BIBLIOGRAPHY}

[1] https://www.iea.org (accessed 19.04.2019)

[2] Schubert, E. Fred. Light - emitting diodes. Wiley Encyclopedia of Electrical and Electronics Engineering, 1999, p. 1-10.

[3] Winston, Roland, Miñano, Juan C. and Benitez, Pablo G. "Nonimaging Optics". Elsevier Academic Press, New York, 2005. ISBN-13: 978-0127597515

[4] Cassarly, William J. and Hayford, Michael, "Illumination optimization: the revolution has begun". Proc. SPIE Vol.4832, p.258269 (2002). https://doi.org/10.1117/12.486466

[5] Koshel, R. John, "Illumination Engineering: Design with nonimaging optics", Wiley IEEE press 2013. DOI: $\underline{10.1002 / 9781118462539}$

[6] Koshel, R. John, "Simplex optimization method for illumination design", Optics Letters Vol.30(6), 649-651 (2005). https://doi.org/10.1364/OL.30.000649

[7] Álvarez Fernández-Balbuena et al. "Application of dynamic merit function to nonimaging systems optimization", Optical Engineering Vol.54 (2), 1-7, 2015. DOl: 10.1117/1.OE.54.2.025107.

[8] Welford, Walter Thompson, Winston, Roland, "High Collection Nonimaging Optics", Academic press, San Diego, 1989. https://doi.org/10.1016/B978-0-12-742885-7.X5001-3

[9] A. Gershun, "The light Field", J. Math. Physics, vol. XVIII, 51-151, 1939.

[10] García-Botella, Ángel, Jiang, Lun, Winston, Roland, "'Optical simulation-based on flowline method". Proc. SPIE Vol.10758, Nonimaging Optics: Efficient Design for Illumination and Solar Concentration XV, 2018. doi: 10.1117/12.2500931

[11] https://ec.europa.eu/energy/en/topics/energy-efficiency/energy-performance-of-buildings

[12] Edwards, L.; Torcelllini, P. Literature review of the effects of natural light on building occupants. National Renewable Energy Lab., Golden, CO.(US), 2002. https://www.doi.org/10.2172/15000841

[13] Mayhoub, M. S.; Carter, D. J. The costs and benefits of using daylight guidance to light office buildings. Building and Environment, 2011, Vol.46, no 3, p. 698-710. https://doi.org/10.1016/j.buildenv.2010.09.014

[14] CIE 173:2012 Tubular daylight guidance system ISBN 9783902842404.

[15] Daniel Vázquez-Moliní et al., "Horizontal daylighting system for office buildings", Energy and Buildings, Vol.67, 2013, p. 525530, https://doi.org/10.1016/i.enbuild.2013.08.040

[16] Omar, Osama, et al. Optimization of daylight utilization in energy saving application on the library in faculty of architecture, design and built environment, Beirut Arab University. Alexandria Engineering Journal, 2018, Vol.57, no 4, p. 3921-3930. https://doi.org/10.1016/i.aej.2018.10.006

[17] García-Fernández, Berta, et al. Light losses in hollow, prismatic light guides related to prism defects: a transmittance model. Chinese Optics Letters, 2015, Vol.13, no 9, p. 092201. http://dx.doi.org/10.3788/COL201513.092201

[18] 2301 OLF Data sheet, Available at: http://multimedia.3m.com/mws/media/11326830/3mtm-optical-lighting-film2405.pdf?fn=OLF\%202405 Tech\%20Data Rev\%20B.pdf

[19] TracePro® Opto-Mechanical Design Software www.lambdares.com

[20] Luminit optics. https://www.luminitco.com/ (Last visited April 2019)

[21] Vazquez-Molini, D., Fernandez-Balbuena, A. A., Garcia-Fernandez, B., \& Bernabeu, E. (2008, September). High efficiency 90 elbow for light guides. In Nonimaging Optics and Efficient Illumination Systems V (Vol. 7059, p. 70590S). International Society for Optics and Photonics. https://doi.org/10.1117/12.796153

[22] D. Vázquez, A. Alvarez, H. Canabal, A. Garcia, S. Mayorga, C. Muro, T. Galan, "Point to point multispectral light projection applied to cultural heritage," Proc. SPIE 10379, Nonimaging Optics: Efficient Design for Illumination and Solar Concentration XIV, 103790K (7 September 2017); doi: 10.1117/12.2281482

[23] D. Vázquez Moliní, A. Álvarez Fernández-Balbuena, B. García-Fernández, P Belloni, "Mathematical model for optimizing damage and color fidelity in museum illumination", Proceedings of LICHT 2016, Karlsruhe, pp. 545-553. 